\title{
On the Use of Geophysical Parameters for the Top-of-Atmosphere Shortwave Clear-Sky Radiance-to-Flux Conversion in EarthCARE
}

\author{
F. TORNOW \\ Institute for Space Sciences, Freie Universität Berlin, Berlin, Germany \\ C. DOMENECH \\ GMV, Madrid, Spain \\ J. FISCHER \\ Institute for Space Sciences, Freie Universität Berlin, Berlin, Germany
}

(Manuscript received 10 June 2018, in final form 18 January 2019)

\begin{abstract}
We have investigated whether differences across Clouds and the Earth's Radiant Energy System (CERES) top-of-atmosphere (TOA) clear-sky angular distribution models, estimated separately over regional $\left(1^{\circ} \times 1^{\circ}\right.$ longitude-latitude) and temporal (monthly) bins above land, can be explained by geophysical parameters from Max Planck Institute Aerosol Climatology, version 1 (MAC-v1), ECMWF twentieth-century reanalysis (ERA-20C), and a MODIS bidirectional reflectance distribution function (BRDF)/albedo/nadir BRDFadjusted reflectance (NBAR) Climate Modeling Grid (CMG) gap-filled products (MCD43GF) climatology. Our research aimed to dissolve binning and to isolate inherent properties or indicators of such properties, which govern the TOA radiance-to-flux conversion in the absence of clouds. We collocated over seven million clear-sky footprints from CERES Single Scanner Footprint (SSF), edition 4, data with above geophysical auxiliary data. Looking at data per surface type and per scattering direction-as perceived by the broadband radiometer (BBR) on board Earth Clouds, Aerosol and Radiation Explorer (EarthCARE)—we identified optimal subsets of geophysical parameters using two different methods: random forest regression followed by a permutation test and multiple linear regression combined with the genetic algorithm. Using optimal subsets, we then trained artificial neural networks (ANNs). Flux error standard deviations on unseen test data were on average $2.7-4.0 \mathrm{~W} \mathrm{~m}^{-2}$, well below the $10 \mathrm{~W} \mathrm{~m}^{-2}$ flux accuracy threshold defined for the mission, with the exception of footprints containing fresh snow. Dynamic surface types (i.e., fresh snow and sea ice) required simpler ANN input sets to guarantee mission-worthy flux estimates, especially over footprints consisting of several surface types.
\end{abstract}

\section{Introduction}

In the absence of clouds, Earth's surface and atmospheric constituents (i.e., absorbing gases and scattering and absorbing aerosols) govern the angular distribution of backscattered solar radiation perceived at top of atmosphere (TOA). Any unforeseen spatial or temporal variations of the surface's appearance (such as the surface roughness of a water body or the vegetation state over land) as well as atmospheric composition (such as aerosol plumes or ozone variations) may translate into an uncertainty when converting an

Corresponding author: Florian Tornow, florian.tornow@ fu-berlin.de observed solar radiance $L$ into corresponding hemispheric solar flux $E$.

An angular distribution model provides a conversion factor, often referred to as anisotropy $\mathbb{R}$, specifically for an observation's viewing-illumination geometry (i.e., solar zenith angle $\theta_{S}$, relative azimuth angle $\varphi$, and viewing zenith angle $\theta_{V}$ ) and an underlying surfaceatmosphere composition (denoted through subscript $c$ ), as shown in Eq. (1):

$$
E\left(\theta_{S}\right)=\frac{L\left(\theta_{S}, \theta_{V}, \varphi\right) \pi}{\mathbb{R}_{c}\left(\theta_{S}, \theta_{V}, \varphi\right)}
$$

The gold standard of angular distribution models has been provided by the Clouds and the Earth's Radiant 
Energy System (CERES) team (Su et al. 2015a) and is widely applied to other satellite missions performing broadband radiometry (Viollier et al. 2009; Dewitte et al. 2008). The upcoming ESA-JAXA mission Earth Clouds, Aerosol and Radiation Explorer (EarthCARE; Illingworth et al. 2015), to be launched in 2021, will be equipped with a broadband radiometer (BBR; e.g., Wallace et al. 2009; Caldwell et al. 2017) observing nadir as well as along track forward and backward (both at $55^{\circ}$ viewing zenith angle). Apart from monitoring TOA fluxes, the mission will conduct a radiative closure assessment, comparing BBR-derived longwave (LW) and shortwave (SW) flux estimates with simulated fluxes. Broadband simulations will act on active-passive retrievals of cloud and aerosol properties for $\sim 1-\mathrm{km}^{2}$ nadir columns (Barker et al. 2011). Agreement between measurement and simulation-based fluxes (within $10 \mathrm{~W} \mathrm{~m}^{-2}$ over $100-\mathrm{km}^{2}$ horizontal assessment domains) translates into confirmed understanding of cloud-aerosolradiation interaction and, ultimately, into trusting simulated vertical heating rate profiles. In the closure assessment, clear-sky scenes will pose less of a challenge. However, simulation biases-arising, for example, from poorly characterized surface or aerosol spectral properties - could easily be identified in a comparison to BBR-derived clear-sky fluxes.

To account for the complex nature of bidirectional reflectance distribution over clear-sky scenes, the CERES team built regional (resolved at $1^{\circ} \times 1^{\circ}$ latitudelongitude cells) and temporal (resolved by calendar month) angular distribution models over land surfaces [CERES Single Scanner Footprint (SSF), edition 4; Su et al. 2015a]. In this study, we investigate whether geophysical variables, that will be available during the EarthCARE mission and either match the data employed to construct CERES angular distribution models (ADMs) or extend the list, could help to dissolve regional and temporal binning. We collocate several datasets quantifying bottom-of-atmosphere (BOA) anisotropy and factors presumed to drive BOA or TOA anisotropy with CERES instantaneous observations. We identify essential variable subsets for each surface type and scattering direction, using two different statistical methods that allow for an efficient exploration of potential variable combinations: multiple linear regression combined with the genetic algorithm, and random forest regression followed by a permutation test. Once identified, optimal variable subsets and CERES TOA SW anisotropies serve to train radiance-to-flux converting artificial neural networks (ANNs). We assess their performance on unseen test samples and, finally, propose modifications for operational application during the EarthCARE mission.
The second section explains datasets, their division into training and test sets, variable selection methods, and the design of ANNs. The third section presents results, and the fourth section discusses findings.

\section{Data and methodology}

\section{a. CERES SSF, edition 4}

We obtained instantaneous TOA SW anisotropies $\mathbb{R}$ from CERES SSF (TOA/Surface Fluxes and Clouds), edition 4, along with parameters on viewing and illumination geometry (i.e., $\theta_{S}, \varphi$, and $\theta_{V}$ ) as well as International Geosphere-Biosphere Programme (IGBP) types and their fraction within each footprint. Included Moderate Resolution Imaging Spectroradiometer (MODIS) cloud fraction served to filter out clear-sky footprints (i.e., $\leq 0.1 \%$ cloud fraction), and $\theta_{V}$ was used to select BBR-like viewing geometries (i.e., $\theta_{V} \leq 2.5^{\circ}$ and $52.5^{\circ} \leq \theta_{V} \leq 57.5^{\circ}$ ).

Anisotropy estimation over clear-sky scenes is thoroughly described in Su et al. (2015a). In short, ADMs over water surfaces were generated per interval of prevailing MODIS-based aerosol optical depth (AOD) and aerosol fine-mode fraction, as well as $10-\mathrm{m}$ wind speed, provided by GEOS-5. Sun-glint-affected geometries were handled separately. ADMs over land surfaces were established individually for each grid box $\left(1^{\circ}\right.$ latitude by $1^{\circ}$ longitude) and calendar month. Intervals of MODISbased normalized density vegetation index (NDVI) and surface elevation variability received further individual treatment. ADMs over snow and ice surfaces were produced based on intervals of respective surface type fraction as well as bright and dark categories. Table 1 lists details on extracted footprints from both Aqua and Terra missions during rotating azimuth plane scan (RAPS) and cross-track mode.

\section{b. Collocated geophysical data}

This study aims to prepare clear-sky TOA SW anisotropy prediction during the future EarthCARE mission. There are two main reasons to investigate the predictability of CERES anisotropies through geophysical parameters. First, to account for a different availability of parameters during the mission compared to what CERES used (see section 2a): for example, meteorological data will be provided by the European Centre for Medium-Range Weather Forecasts (ECMWF; delivering forecast data, coined X-MET) instead of the GEOS-5, and imager-based AOD will be avoided (to minimize dependencies of the radiance-to-flux conversion on other EarthCARE level 2 products during operations; T. Wehr 2015, personal communication) so 
TABLE 1. A list of extracted CERES footprints, obtained after filtering for clear-sky and BBR-like conditions-in total 7.3 million footprints.

\begin{tabular}{lccc}
\hline \hline & \multicolumn{2}{c}{ No. of CERES footprints } & \\
\cline { 2 - 3 } Year & $\begin{array}{c}\text { Terra (FM1 } \\
\text { and FM2) }\end{array}$ & $\begin{array}{c}\text { Aqua }(\mathrm{FM} 3 \\
\text { and FM4) }\end{array}$ & Mode \\
\hline 2000 & 108828 & - & RAPS \\
2001 & 208492 & - & RAPS \\
2002 & 207365 & 67024 & RAPS \\
2003 & 222016 & 208360 & RAPS \\
2004 & 215843 & 274869 & RAPS \\
2005 & 25655 & 25224 & RAPS \\
2006 & - & - & - \\
2007 & 2892051 & 2819907 & Cross track \\
\hline
\end{tabular}

that other sources need to be considered. Second and the main focus of this study, the CERES approach produced separate ADMs over land surfaces per grid box and per calendar month, in order to account for the variable appearance of land across space and time. To dissolve regional and temporal binning of CERES ADMs (see section 2a) and still reach a comparable uncertainty in TOA flux estimation during the EarthCARE mission, we rely on additional geophysical variables. The most useful variables quantify BOA or even TOA anisotropy directly. Variables that indicate scattering or absorption effects in the surface-atmosphere system and characterize clear-sky TOA anisotropy indirectly are also considered useful. All footprints listed in Table 1 were spatially and temporally collocated with following datasets, either matching or substituting the variables employed by CERES or extending the list.

Via the nearest neighbor approach, we extracted median total AOD from the Max Planck Institute Aerosol Climatology, version 1 (MAC-v1; monthly fields at $1^{\circ}$ resolution in longitude and latitude; Kinne et al. 2013), which is a fusion of ground-based sunphotometer measurements and a globally modeled aerosol background. However, fine-mode fraction or other variables indicating aerosol type, size distribution, or optical properties were not included in version 1 . Also via nearest neighbor, we obtained a selection of atmospheric and surface parameters from the ECMWF twentieth-century reanalysis (ERA-20C; using the years 2000-07; Poli et al. 2016). Fields were downloaded at 3hourly and T159 spatial resolution. To match CERES variables, we extracted $10-\mathrm{m}$ wind speed, low- and highvegetation leaf area index [LAI; here used as substitute for NDVI, and also used as input to vegetationscattering models, e.g., by Ross (1981)], and land surface roughness (as substitute for CERES surface elevation variability). To extend the list, we further selected sea surface roughness (referred to as Charnock;
Charnock 1955), total column amounts of atmospheric ozone and water vapor, snow depth, and sea ice fraction.

Based on 12 years (2002-13) of MODIS bidirectional reflectance distribution function (BRDF)/albedo/nadir BRDF-adjusted reflectance (NBAR) Climate Modeling Grid (CMG) gap-filled products (MCD43GF; Moody et al. 2008), we use a climatology (Z. Qu 2015, personal communication) of RossThick-LiSparse kernel weights that serves EarthCARE level-2 processors. Kernels are thoroughly described in Lucht et al. (2000). In short, under the assumption of dense (or thick) leaf canopy and sparsely ensembled geometric objects, kernels describe volumetric $\left(K_{\mathrm{vol}}\right)$, geometric $\left(K_{\mathrm{geo}}\right)$, and isotropic bidirectional reflectance distribution effects. Respective weights $\left(f_{\mathrm{vol}}, f_{\text {geo }}\right.$, and $\left.f_{\text {iso }}\right)$ semiempirically and linearly combine all kernels. We collocated kernel weights (provided at $1-\mathrm{km}^{2}$ spatial resolution) according to each CERES footprint's field of view and resulting point spread function (detailed description in Wielicki et al. 1996). From kernel weights in visible (VIS; 0.3-0.7 $\mu \mathrm{m}$ ) and near-infrared (NIR; $0.7-5.0 \mu \mathrm{m})$ spectra, we calculated surface reflectances [Eq. (2), shown for VIS reflectance $r^{\mathrm{VIS}}$ ] and albedos for both black- and white-sky conditions [Eq. (3), shown for VIS white-sky albedo $\left.a_{w}^{\mathrm{VIS}}\right]$. Finally, we produced BOA anisotropies, $R_{w}^{\mathrm{VIS}}, R_{b}^{\mathrm{VIS}}, R_{w}^{\mathrm{NIR}}$, and $R_{b}^{\mathrm{NIR}}$, corresponding to each footprint's viewing and illumination geometry [Eq. (4), shown for VIS whitesky anisotropy $\left.R_{w}^{\mathrm{VIS}}\right]$ :

$$
\begin{aligned}
r^{\mathrm{VIS}} \pi & =f_{\text {iso }}^{\mathrm{VIS}}+f_{\mathrm{vol}}^{\mathrm{VIS}} K_{\mathrm{vol}}\left(\theta_{S}, \theta_{V}, \varphi\right)+f_{\mathrm{geo}}^{\mathrm{VIS}} K_{\mathrm{geo}}\left(\theta_{S}, \theta_{V}, \varphi\right), \\
a_{w}^{\mathrm{VIS}} & =f_{\text {iso }}^{\mathrm{VIS}}+0.189184 f_{\mathrm{vol}}^{\mathrm{VIS}}-1.377622 f_{\mathrm{geo}}^{\mathrm{VIS}} \\
R_{w}^{\mathrm{VIS}} & =\frac{r^{\mathrm{VIS}} \pi}{a_{w}^{\mathrm{VIS}}}
\end{aligned}
$$

All collocated parameters and their spatiotemporal resolution are summarized in Table 2. We also computed two bidirectional reflectance effects. The hotspot effect $1+F(G)$ was calculated as shown in Rahman et al. (1993) and uses MCD43GF-based VIS surface reflection under zenith illumination and zenith viewing conditions $r_{0}$, as well as instantaneous viewing and illuminations angles:

$$
1+F(G)=1+\frac{1-r_{0}}{1+G}
$$

where

$$
G=\left(\tan ^{2} \theta_{S}+\tan ^{2} \theta_{V}-2 \tan \theta_{S} \tan \theta_{V} \cos \varphi\right)^{1 / 2},
$$


TABLE 2. A list of collocated geophysical parameters to each CERES footprint, listed in Table 1.

\begin{tabular}{|c|c|c|c|}
\hline Source & Parameter & $\begin{array}{l}\text { Spatial and temporal } \\
\text { resolution }\end{array}$ & Collocation type \\
\hline ERA-20C (Poli et al. 2016) & $\begin{array}{l}\text { Total column ozone } \\
\text { Total column water vapor } \\
\text { Leaf area index high vegetation } \\
\text { Leaf area index low vegetation } \\
10-\mathrm{m} U \text { wind component } \\
10-\mathrm{m} V \text { wind component } \\
\text { Forecast surface roughness } \\
\text { Charnock } \\
\text { Snow depth } \\
\text { Sea ice cover }\end{array}$ & 125 km (T159); 3 hourly & Nearest neighbor \\
\hline MAC-v1 (Kinne et al. 2013) & Median total AOD & $1^{\circ}$ lat-lon; monthly & Nearest neighbor \\
\hline $\begin{array}{l}\text { MCD43GF (Moody et al. } \\
\text { 2008) (a climatology based } \\
\text { on the years 2002-13; Z. } \\
\text { Qu 2015, personal } \\
\text { communication) }\end{array}$ & $\begin{array}{l}\text { VIS isotropic model parameter } f_{\text {iso }}^{\mathrm{VIS}} \\
\text { VIS volume-scattering model parameter } f_{\mathrm{vol}}^{\mathrm{VIS}} \\
\text { VIS geometric-scattering model parameter } f_{\text {geo }}^{\mathrm{VIS}} \\
\text { NIR isotropic model parameter } f_{\text {iso }}^{\mathrm{NIR}} \\
\text { NIR volume-scattering model parameter } f_{\mathrm{vol}}^{\mathrm{NIR}} \\
\text { NIR geometric-scattering model parameter } f_{\text {geo }}^{\mathrm{NIR}}\end{array}$ & $1 \mathrm{~km} ; 16$ day & $\begin{array}{l}\text { Nearest in time; weighted } \\
\text { areal average according to } \\
\text { CERES instantaneous PSF }\end{array}$ \\
\hline
\end{tabular}

$r_{0}=\left[f_{\text {iso }}^{\mathrm{VIS}}+f_{\mathrm{vol}}^{\mathrm{VIS}} K_{\mathrm{vol}}(0,0, \varphi)+f_{\mathrm{geo}}^{\mathrm{VIS}} K_{\mathrm{geo}}(0,0, \varphi)\right] / \pi$

The sun-glint reflectance $r_{g}$ (Cox and Munk 1954) was computed as shown in Wald and Monget (1983) and essentially uses ERA-20C $10-\mathrm{m}$ wind speed $W_{10 \mathrm{~m}}=$ $\left(U_{10 \mathrm{~m}}^{2}+V_{10 \mathrm{~m}}^{2}\right)^{1 / 2}$, the Fresnel reflection factor $\rho(\omega)$ for a perfectly smooth surface, and above instantaneous illumination and viewing angles:

$$
r_{g}=\frac{\pi \rho(\omega) P\left(\theta_{n}, W_{10 \mathrm{~m}}\right)}{4 \cos \theta_{S} \cos \theta_{V} \cos ^{4} \theta_{n}},
$$

where

$$
\begin{aligned}
P\left(\theta_{n}, W_{10 \mathrm{~m}}\right) & =\frac{1}{\pi \sigma^{2}} \exp \left(-\frac{\tan ^{2} \theta_{n}}{\sigma^{2}}\right), \\
\sigma^{2} & =0.003+0.00512 W_{10 \mathrm{~m}}, \\
\theta_{n} & =\arccos \left(\frac{\cos \theta_{V}+\cos \theta_{S}}{2 \cos \omega}\right), \\
\cos 2 \omega & =\cos \theta_{V} \cos \theta_{S}+\sin \theta_{V} \sin \theta_{S} \cos \varphi .
\end{aligned}
$$

\section{c. Division into training and test datasets}

The resulting radiance-to-flux conversion should be generalized; that is, it should achieve an analogous performance on unseen or future data of a similar nature. Following this study, such data will originate from EarthCARE-based measurements and retrievals as well as acquired auxiliary data, comparable to parameters presented in sections $2 \mathrm{a}$ and $2 \mathrm{~b}$. To test the ability of the following methods to generalize, we hold back a random $25 \%$ portion of data (referred to as test data) until final evaluation. We assume that the difference between predictions using the test data and the original CERES flux estimates (referred to as test error) reflects the performance on any future data. The remaining $75 \%$ (referred to as training data) is used to perform variable selection and to optimize and train ANN regression methods. As shown in sections $2 \mathrm{~d}$ and $2 \mathrm{e}$, some model parameters (e.g., the number of hidden neurons in an ANN) require adjustment to function optimally. To choose the optimal adjustment, a validation dataset taken from the training dataset serves to initially assess the performance on unused data. To incorporate the full range of training samples, cross validation repeatedly uses a random portion of the training data as validation set, using remaining samples for actual training.

\section{d. Variable selection}

When selecting variables, one generally attempts to identify the optimal subset $\Gamma \in X=\left\{x_{1}, \ldots, x_{N}\right\}$ of regressors (also referred to as independent parameters where $X$ denotes the full set of parameters) to predict the regressand $y$ (also known as the dependent parameter, in this study TOA SW anisotropy $\mathbb{R}$ ). Using singlesurface footprints of the training dataset (about two-thirds of the training samples), we performed variable selection in two different ways. Both methods explore the parameter space efficiently (i.e., trying out as few parameter combinations as necessary, and avoiding as many dispensable combinations as possible), and relate to anisotropy either linearly or nonlinearly. 
First, we applied the nonlinear random forest regression [thoroughly described in Breiman (2001)]. In short, a random forest consists of $T$ decision trees (here $T=25$ ). Each tree relies on geophysical parameters for decision split nodes and provides a constant predicted value $\tilde{y}_{t}$ at the end of each branch. Hence, a tree's $t$ predicted value is $\tilde{y}_{t}=f\left(\Gamma_{\mathrm{DT} ; t}\right)=\tilde{y}_{t} \mid \Gamma_{\mathrm{DT} ; t}$, where $\Gamma_{\mathrm{DT} ; t}$ is the subset of parameters used by an individual tree. The prediction $\tilde{y}$ of the entire random forest is the arithmetic mean over predictions from all trees $\tilde{y}_{1}, \ldots, \tilde{y}_{T}$. To train the random forest, repeatedly drawn random subsets of regressors were searched in several rounds for optimum split nodes to further refine each tree's structure and ultimately reduce errors of predictions. To identify the essential subset of the trained random forest $\Gamma_{\mathrm{RF}}$ (i.e., the collection of employed parameters of $\Gamma_{\mathrm{DT} ; 1}$ through $\Gamma_{\mathrm{DT} ; T}$ ), we then used a permutation test (Strobl and Zeileis 2009) that goes through each regressor $x_{i}$ one by one. The test measures the increase in root-mean-square deviation $\Delta$ RMSD of predictions on each tree's set $\Gamma_{\mathrm{DT} ; t}^{x_{i}}$ with regressor $x_{i}$ permutated (if part of the set) against the original set $\Gamma_{\mathrm{DT} ; t}$ with nonpermutated regressors over $M$ random validation samples (here $M=1000$ ), as shown in Eq. (13). Generally, an irrelevant regressor should produce $\triangle \mathrm{RMSD}=0$, while an important one (providing at least one important split node in at least one tree) should give $\triangle$ RMSD $>0$ :

$$
\begin{aligned}
\Delta \operatorname{RMSD}\left(x_{i}\right)= & \sqrt{\frac{\sum_{m=1}^{M}\left(\left[\frac{1}{T} \sum_{t=1}^{T} \tilde{y}_{t} \mid \Gamma_{\mathrm{DT} ; t}^{x_{i}}(m)\right]-y(m)\right)^{2}}{M-1}} \\
& -\sqrt{\frac{\sum_{m=1}^{M}\left(\left[\frac{1}{T} \sum_{t=1}^{T} \tilde{y}_{t} \mid \Gamma_{\mathrm{DT} ; t}(m)\right]-y(m)\right)^{2}}{M-1}} .
\end{aligned}
$$

Repeated 100 times, we identified parameters as part of the optimal subset that had a distribution of $\triangle$ RMSD significantly larger (one-sided $t$ test with $p<0.1$ ) than the $\triangle$ RMSD of a known irrelevant variable. We assume these parameters have meaningfully contributed to the construction of the random forest and the regression.

Second, we used the genetic algorithm wrapping multiple linear regression (described in, e.g., Mehmood et al. 2012) to build a linear model $\tilde{y}=f\left(\Gamma_{\mathrm{LM}}\right)=\Gamma_{\mathrm{LM}}^{\mathrm{T}} w$, where $\Gamma_{\mathrm{LM}}$ is the optimal subset of geophysical parameters for a linear model (superscript $\mathrm{T}$ indicates its transpose) and $w$ is the least squares weight to approximate $\tilde{y}$, here TOA SW anisotropy $\mathbb{R}$. The optimal subset $\Gamma_{\mathrm{LM}}$ is identified using a survival-of-the-fittest method, inspired by biological evolution theory. Starting with an initial population of linear models - each with a random binary string of length $N$ indicating participation of each regressor $x_{i}$ (" 0 " or " 1 " to exclude or include from the linear model, respectively)-fitter models are more likely to survive into the next round. Fitness is evaluated through a fitness function (here the Bayesian information criterion). From all survivors, new linear models can be added to the population through mutation (i.e., the random switch of a bit from 0 to 1 , or vice versa, in the binary string of a surviving model) or crossover (i.e., the random unification of two surviving binary strings from two models). The newly generated population is reevaluated for fitness to determine new survivors, and so on. In the final round the fittest linear model is presumed to consist of set $\Gamma_{\mathrm{LM}}$. In this study, we identified subsets $\Gamma_{\mathrm{LM} ; 1}$ through $\Gamma_{\mathrm{LM} ; 100}$ from 100 repetitions [each using a population size of 500, the Bayesian information criterion fitness function, linear ranking-based roulette wheel selection, a mutation probability of 0.01 , a crossover probability of 0.95 , and an elitism of 3 over maximum 50 iterations as defined in Scrucca (2013)]. We selected the subset which performed best [i.e., had the lowest RMSD, as shown in Eq. (14)] in a cross validation (using $25 \%$ of the training data) of size $M$ :

$$
\Gamma_{\mathrm{LM}}=\arg \min \left[\operatorname{RMSD}\left(\Gamma_{\mathrm{LM} ; 1}\right), \ldots, \operatorname{RMSD}\left(\Gamma_{\mathrm{LM} ; 100}\right)\right],
$$

where

$$
\operatorname{RMSD}\left(\Gamma_{\mathrm{LM} ; i}\right)=\sqrt{\frac{1}{M-1} \sum_{m=1}^{M}\left[\Gamma_{\mathrm{LM} ; i}^{\mathrm{T}}(m) w-y(m)\right]^{2}} .
$$

\section{e. Artificial neural networks}

ANNs are nonlinear regression tools that require relatively few model parameters. They are therefore a suitable regression method to perform the radiance-toflux conversion during the mission. Inspired by the biological neural pathways in brains, regression with ANNs aims to connect an input signal (here a subset of geophysical regressors $X$ ) to a learned output (here TOA SW anisotropy $\mathbb{R}$ ). Such connections (resembling biological synapses) are usually not directly connecting in- and output, but involve at least one intermediate layer (referred to as hidden layer) of nodes (i.e., artificial neurons)-each receiving potential contribution from any neuron of the previous layer (the input layer in cases with only one hidden layer). Through a transfer function (e.g., tanh) the weighted sum of these contributions at each node provides modulated input to the follow-up 
layer (the output layer in cases with only one hidden layer). Weights (corresponding to synapse strength) are learned through training, in this study through the back-propagation algorithm. The resulting nonlinear regression makes it possible to approximate progressively complex relations with a growing number of hidden layers. All ANNs in this study consisted of a single hidden layer and were trained using the training set (see section 2c). Both input and output were standardized before training. To find the optimal number of hidden neurons for each ANN, we repeated training for odd numbers of hidden neurons from 3 to $2 J-1$ (where $J$ is the number of input parameters) and selected the best performer via cross validation (using $33 \%$ of the training data).

\section{Results}

To examine whether geophysical auxiliary parameters could explain variations in CERES TOA anisotropy across regional and temporal models, we collocated over seven million instantaneous clear-sky CERES footprints (described in section 2a) with datasets that either substitute CERES-employed parameters during the EarthCARE mission, directly quantify BOA anisotropy, or indirectly relate to scattering and absorption effects involving surface and atmosphere under clearsky conditions: an MCD43GF climatology (providing bottom-of-atmosphere anisotropy information over land surfaces), the MAC-v1 AOD climatology, and ERA-20C reanalysis data (providing information on atmospheric gases and numerous parameters relevant to the state of the surface) - all introduced in more detail in section 2b. In preparation for the EarthCARE mission, we focused on nadir-viewing $\left(\theta_{V} \sim 0^{\circ}\right)$ and off-nadir-viewing (off-nadir forward: $\theta_{V} \sim 55^{\circ}$, and $\varphi<90^{\circ}$ or $\varphi>270^{\circ}$; off-nadir backward: $\theta_{V} \sim 55^{\circ}, 90^{\circ} \leq \varphi \leq 270^{\circ}$ ) radiance measurements only.

Section 3a demonstrates the capability of various parameters, such as leaf area index, to serve as predictors for TOA anisotropy and introduces the separate treatment of surface types and scattering directions. In section $3 b$ we present optimal parameter subsets for TOA anisotropy estimation-identified through two feature selection methods-and then assess the performance of optimal subsets when used as input to radiance-to-flux converting ANNs. Finally, section 3c presents limitations and adaptations of this methodology for the future EarthCARE mission. While sections $3 a$ and $3 b$ focus on CERES footprints that consist entirely of a single-surface type (about twothirds of all samples), section $3 \mathrm{c}$ considers mixedsurface footprints.

\section{a. Exploring the capability of geophysical parameters}

Earth's surface offers a rich set of characteristic appearances, each with a unique bidirectional reflectance distribution. Similar to previous studies (e.g., Domenech and Wehr 2011), we used the IGBP surface classification (Townshend 1992) to separately perform radiance-toflux conversions over forest (IGBP types 1, 2, 3, 4, and 5), fresh snow (19), sea ice (20), savannah (8 and 9), grassland (10,11, 12, and 14), shrubland (6 and 7), desert (13 and 16), permanent snow and ice (15), and open water (17) surfaces.

To introduce and demonstrate how powerful collocated and generated geophysical parameters are for the prediction of TOA SW anisotropy, Fig. 1 shows their application in simple linear models. All footprints shown consist of a single-surface type. Note the initial spread in TOA SW anisotropy ( $y$ axis of the left column of panels)—in part resulting from various illumination and viewing geometries. The hotspot parameter (shown in Fig. 1a over the Saharan desert) accounted for geometries and partly explained TOA anisotropy variations. Based on a climatology of the MCD43GF product, BOA VIS white-sky anisotropy (Fig. 1b) further explained resulting residuals. The VIS black-sky anisotropy is complementary to the VIS white-sky anisotropy (Figs. $1 \mathrm{~g}$ and $1 \mathrm{~h}$ ).

Climatological surface anisotropies might fail to account for interannual or nonseasonal changes of land surface characteristics. ERA-20C data could account for this, for example, through the LAI (shown in Fig. 1i), potentially serving as a proxy for enhanced volumetric scattering.

Atmospheric gases absorb parts of the solar spectrum, affecting off-nadir views more than nadir views through respective atmospheric pathlengths. Total column ozone explained some anisotropy residuals (shown in Fig. 1f). Aerosols may also scatter and redistribute solar radiation within the upward hemisphere. As noted in section $2 \mathrm{~b}$, we decided to use an AOD climatology. The MAC-v1 climatology did not include single-scattering albedo (or aerosol type). However, through a surfacespecific application of AOD, we potentially relate responses in TOA SW anisotropy to varying levels of surface-specific aerosol mixtures. Figures 1c and 1e show examples of explained anisotropy residuals.

\section{b. Finding optimal subsets}

Extracted geophysical parameters are applicable to the prediction of TOA SW anisotropy, as demonstrated in section 3a. For operational application during the mission, ANNs offer nonlinear regression with relatively few model parameters (section 2e) and are therefore 


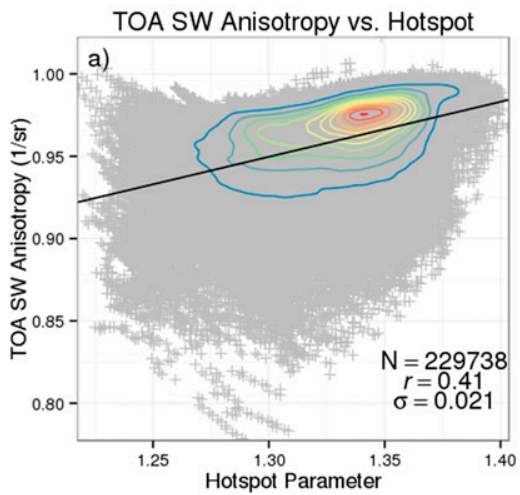

TOA SW Anisotropy vs. MODIS BOA Aniso.

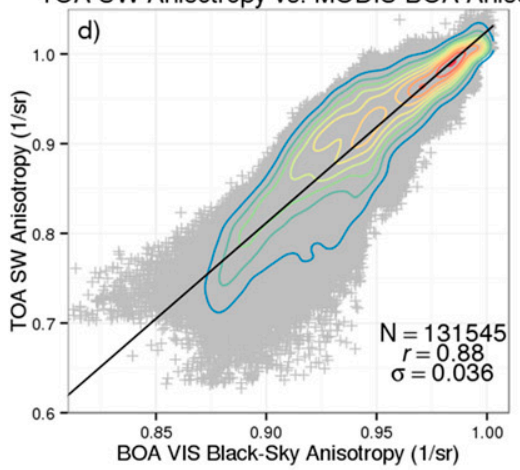

TOA SW Anisotropy vs. MODIS BOA Aniso.

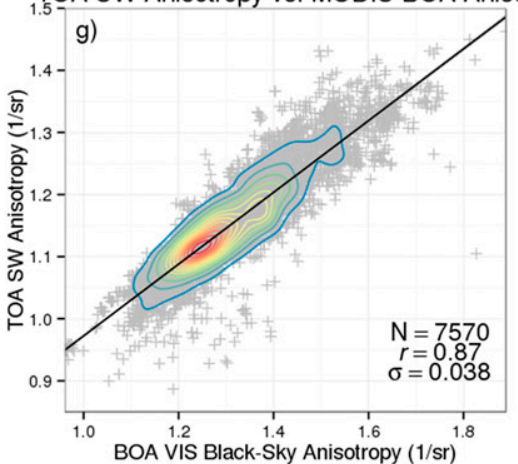

Saharan Desert Surfaces

Backward Scattered Residuals vs. MODIS BOA Anisotropy
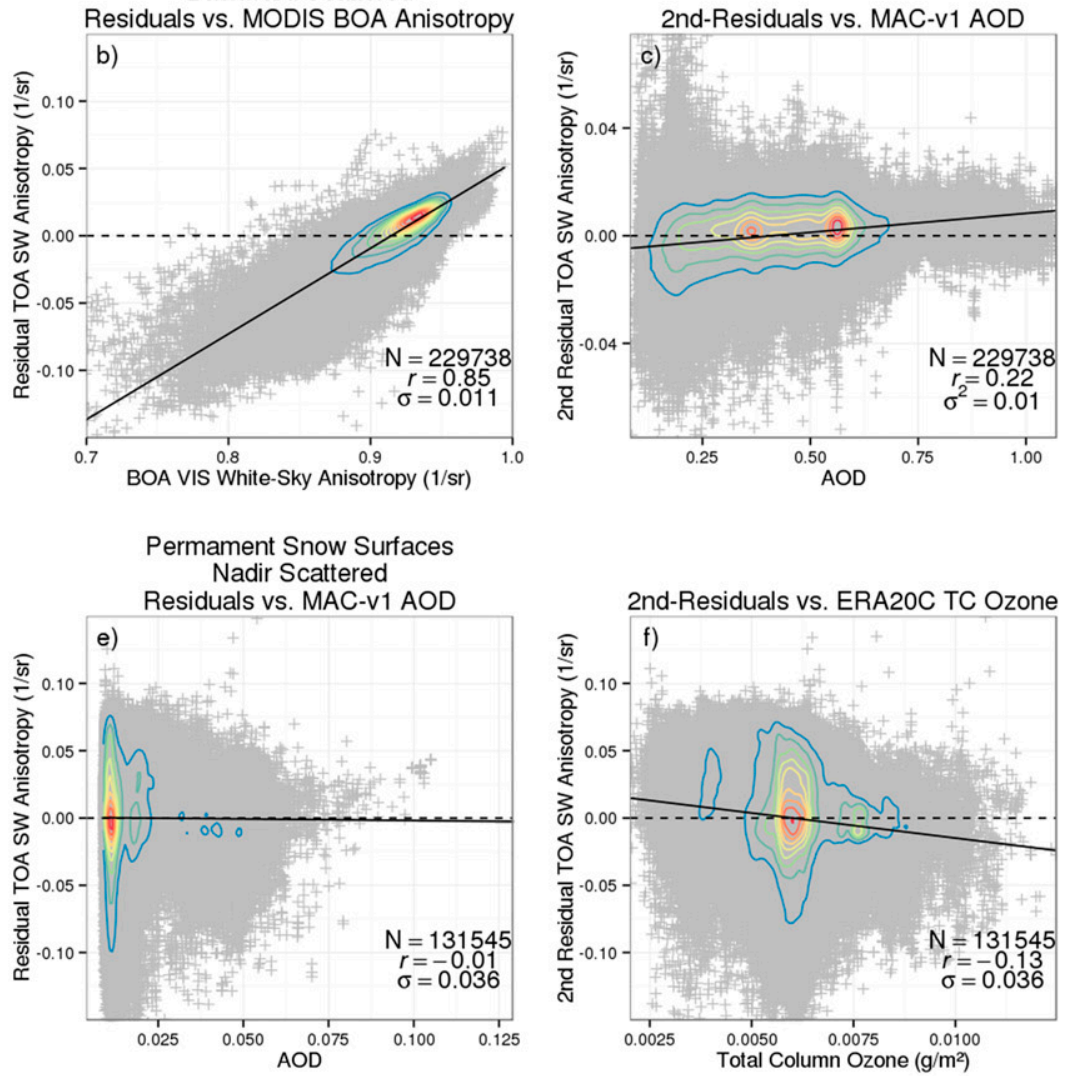

Forest Surfaces

Forward Scattered

Residuals vs. MODIS BOA Anisotropy

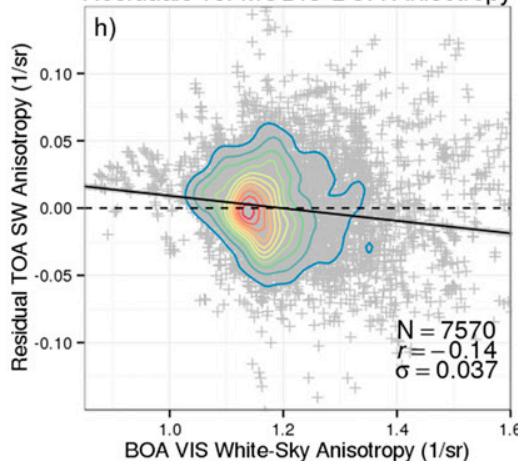

2nd-Residuals vs. ERA20C High Veg. LAI

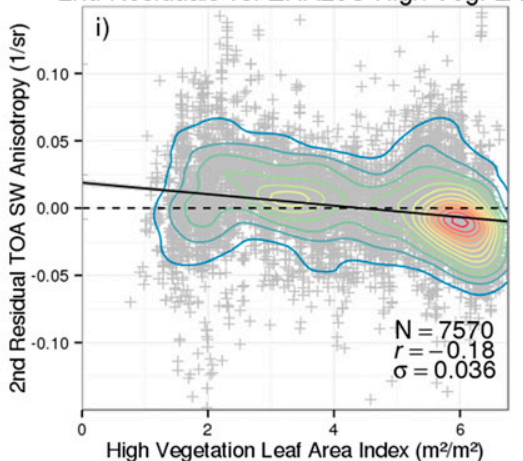

FIG. 1. We present the use of collocated geophysical parameters to explain varying TOA SW anisotropy for three surfaces at particular scattering directions (by row): Saharan desert surfaces at backward scattering $\left(\theta_{V} \sim 55^{\circ}\right.$, and $\varphi<90^{\circ}$ or $\left.\varphi>270^{\circ}\right)$; permanent snow and ice surfaces at nadir scattering $\left(\theta_{V} \sim 0^{\circ}\right)$; and forest surfaces at forward scattering $\left(\theta_{V} \sim 55^{\circ}, 90^{\circ} \leq \varphi \leq 270^{\circ}\right)$. From left to right a simple linear model evolves. (a),(d),(g) A linear fit with an initial parameter leaves residuals, which we aim to explain through an extended linear model using (b),(e),(h) an additional parameter which still leaves residuals. (c),(f),(i) A third parameter extends this linear model further. Colored lines mark the observed probability density (blue to red from low to high). Black lines show simple linear fits.

widely applied to predict TOA SW anisotropy in other missions (as in Domenech and Wehr 2011; Loukachine and Loeb 2004; Capderou and Viollier 2006). Like most other regression methods, ANNs require a careful selection of input parameters. Including useless parameters could add unwanted noise while excluding useful parameters may reduce performance (i.e., the bias-variance dilemma; Theodoridis and Koutroumbas 2009). 
TABLE 3. Results of parameter selections applied to each surface type and BBR-perceivable scattering direction (B: backward; F: forward; N: nadir). Dots and their shape connote selection method: selected by both random forest and the genetic algorithm (•); excluded by both (blank); selected only by random forest (o); and selected only by the genetic algorithm ( $\star$ ); and "/" indicates general exclusion from parameter selection. Sav, Savannah; Grass, Grassland; Shrub, Shrubland; and Perm, Permanent.

\begin{tabular}{|c|c|c|c|c|c|c|c|c|c|c|c|c|c|c|c|c|c|c|c|c|c|c|c|c|c|c|c|}
\hline \multirow{3}{*}{$\begin{array}{c}\text { Parameter//scattering } \\
\text { direction }\end{array}$} & \multicolumn{27}{|c|}{ Surface types } \\
\hline & \multicolumn{3}{|c|}{ Forest } & \multicolumn{3}{|c|}{$\begin{array}{l}\text { Fresh } \\
\text { snow }\end{array}$} & \multicolumn{3}{|c|}{ Sea ice } & \multicolumn{3}{|c|}{ Sav } & \multicolumn{3}{|c|}{ Grass } & \multicolumn{3}{|c|}{ Shrub } & \multicolumn{3}{|c|}{$\begin{array}{l}\text { Perm } \\
\text { snow }\end{array}$} & \multicolumn{3}{|c|}{ Desert } & \multicolumn{3}{|c|}{$\begin{array}{l}\text { Open } \\
\text { water }\end{array}$} \\
\hline & B & $\mathrm{F}$ & $\mathrm{N}$ & B & $\mathrm{F}$ & $\mathrm{N}$ & B & $\mathrm{F}$ & $\mathrm{N}$ & B & $\mathrm{F}$ & $\mathrm{N}$ & B & $\mathrm{F}$ & $\mathrm{N}$ & B & $\mathrm{F}$ & $\mathrm{N}$ & $\mathrm{B}$ & $\mathrm{F}$ & $\mathrm{N}$ & $\mathrm{B}$ & $\mathrm{F}$ & $\mathrm{N}$ & B & $\mathrm{F}$ & $\mathrm{N}$ \\
\hline Normalized radiance & 0 & $\bullet$ & $\star$ & & $\star$ & $\bullet$ & $\star$ & $\bullet$ & $\bullet$ & 0 & $\bullet$ & $\star$ & $\bullet$ & $\bullet$ & $\bullet$ & $\bullet$ & $\bullet$ & $\bullet$ & $\bullet$ & $\bullet$ & $\bullet$ & $\bullet$ & $\bullet$ & $\bullet$ & $\bullet$ & $\bullet$ & $\bullet$ \\
\hline$\theta_{S}$ or $\sin \left(\theta_{S}\right)$ & $\circ$ & $\bullet$ & $\bullet$ & $\circ$ & & $\bullet$ & $\circ$ & $\circ$ & $\bullet$ & $\bullet$ & $\bullet$ & $\bullet$ & $\bullet$ & $\bullet$ & $\bullet$ & $\bullet$ & $\bullet$ & $\bullet$ & $\bullet$ & $\bullet$ & $\bullet$ & $\bullet$ & $\bullet$ & $\circ$ & $\bullet$ & $\bullet$ & $\bullet$ \\
\hline$\theta_{V}$ or $\sin \left(\theta_{V}\right)$ & $\bullet$ & $\bullet$ & & $\bullet$ & $\star$ & & $\bullet$ & $\bullet$ & & $\bullet$ & $\bullet$ & & $\bullet$ & $\bullet$ & & $\bullet$ & $\bullet$ & $\star$ & $\bullet$ & $\circ$ & & $\star$ & $\star$ & & $\bullet$ & $\bullet$ & \\
\hline$\varphi$ or $\sin (\varphi)$ & $\bullet$ & $\bullet$ & I & & $\bullet$ & I & $\bullet$ & $\bullet$ & l & ० & $\bullet$ & I & $\bullet$ & $\bullet$ & l & $\bullet$ & $\bullet$ & / & $\bullet$ & $\bullet$ & l & $\bullet$ & $\bullet$ & l & $\bullet$ & $\bullet$ & l \\
\hline Sun-glint reflectance $r_{g}$ & $\star$ & & $\star$ & & & & $\star$ & $\bullet$ & $\bullet$ & & $\star$ & $\star$ & & $\star$ & $\star$ & & $\star$ & $\star$ & $\star$ & $\star$ & $\star$ & $\star$ & $\star$ & & $\bullet$ & $\bullet$ & $\bullet$ \\
\hline Hot spot effect $1+F(G)$ & $\bullet$ & $\bullet$ & $\bullet$ & $\star$ & $\star$ & $\bullet$ & $\bullet$ & $\bullet$ & $\bullet$ & $\bullet$ & $\bullet$ & $\bullet$ & $\bullet$ & $\bullet$ & $\bullet$ & $\bullet$ & $\bullet$ & $\bullet$ & $\bullet$ & $\bullet$ & $\bullet$ & $\bullet$ & $\bullet$ & $\bullet$ & $\star$ & $\star$ & $\star$ \\
\hline MAC-v1 median total AOD & $\bullet$ & $\bullet$ & & $\star$ & & & $\circ$ & $\circ$ & $\bullet$ & $\bullet$ & $\bullet$ & $\star$ & $\bullet$ & $\bullet$ & $\star$ & $\bullet$ & $\bullet$ & $\bullet$ & $\bullet$ & $\bullet$ & $\bullet$ & $\bullet$ & $\bullet$ & $\bullet$ & $\bullet$ & $\bullet$ & $\star$ \\
\hline ERA-20C ozone & ० & ○ & $\star$ & & & $\star$ & ० & $\bullet$ & $\bullet$ & $\bullet$ & $\circ$ & $\star$ & $\bullet$ & $\circ$ & & $\circ$ & & $\bullet$ & $\bullet$ & $\circ$ & $\bullet$ & & & $\bullet$ & ○ & ○ & $\bullet$ \\
\hline ERA-20C TCWV & $\bullet$ & $\bullet$ & $\star$ & & & & $\bullet$ & $\bullet$ & $\bullet$ & $\bullet$ & ० & $\star$ & $\circ$ & $\star$ & $\star$ & $\bullet$ & & & $\bullet$ & ० & $\bullet$ & $\star$ & $\star$ & $\star$ & ○ & & $\bullet$ \\
\hline MCD43GF-based $R_{b}^{\mathrm{VIS}}$ & $\bullet$ & $\bullet$ & $\circ$ & $\bullet$ & $\star$ & $\circ$ & l & l & l & $\bullet$ & 0 & $\star$ & $\bullet$ & $\bullet$ & ० & $\bullet$ & $\bullet$ & $\bullet$ & $\bullet$ & $\circ$ & $\bullet$ & $\bullet$ & $\bullet$ & $\bullet$ & l & l & / \\
\hline MCD43GF-based $R_{w}^{\mathrm{VIS}}$ & $\circ$ & 0 & $\star$ & $\circ$ & & $\circ$ & l & l & l & $\circ$ & $\bullet$ & $\star$ & $\bullet$ & $\circ$ & $\star$ & $\bullet$ & $\bullet$ & $\bullet$ & $\bullet$ & $\circ$ & $\bullet$ & $\bullet$ & $\bullet$ & $\bullet$ & I & l & I \\
\hline MCD43GF-based $R_{b}^{\mathrm{NIR}}$ & $\bullet$ & $\circ$ & $\bullet$ & $\bullet$ & & $\bullet$ & l & l & l & $\bullet$ & $\bullet$ & $\circ$ & $\bullet$ & $\bullet$ & $\bullet$ & $\bullet$ & $\bullet$ & $\bullet$ & $\bullet$ & $\bullet$ & $\bullet$ & $\bullet$ & $\bullet$ & $\bullet$ & / & / & l \\
\hline MCD43GF-based $R_{w}^{\mathrm{NIR}}$ & $\bullet$ & $\circ$ & $\bullet$ & $\bullet$ & $\star$ & $\bullet$ & l & l & l & $\bullet$ & $\bullet$ & & $\circ$ & $\bullet$ & $\bullet$ & ○ & $\bullet$ & $\bullet$ & $\circ$ & $\bullet$ & $\bullet$ & $\bullet$ & $\bullet$ & $\circ$ & I & / & / \\
\hline ERA-20C LAI low vegetation & $\star$ & & & & & & l & l & l & $\bullet$ & $\bullet$ & $\star$ & $\circ$ & $\circ$ & $\star$ & $\bullet$ & $\star$ & $\star$ & / & l & 1 & $\star$ & $\star$ & & / & / & l \\
\hline ERA-20C LAI high vegetation & $\bullet$ & $\bullet$ & & $\star$ & $\star$ & $\star$ & / & / & / & $\bullet$ & ○ & & & & & $\star$ & & $\star$ & l & / & l & $\star$ & & & / & / & l \\
\hline ERA-20C 10-m wind & & $\circ$ & & & & & $\star$ & $\circ$ & $\bullet$ & $\circ$ & $\star$ & & $\star$ & $\circ$ & & $\bullet$ & & $\star$ & $\bullet$ & $\bullet$ & $\circ$ & & & & $\star$ & $\circ$ & $\circ$ \\
\hline ERA-20C surface roughness & $\bullet$ & o & & $\bullet$ & & $\star$ & & & & $\bullet$ & $\bullet$ & $\star$ & $\bullet$ & $\circ$ & & $\bullet$ & $\circ$ & & $\star$ & & & & & & & & \\
\hline ERA-20C Charnock & & & & & & & & & & & & & & & & & & & & & & & & & & & \\
\hline ERA-20C snow depth & / & l & l & $\star$ & & & l & l & l & l & l & l & l & l & / & / & l & l & & $\star$ & & / & l & l & l & / & l \\
\hline
\end{tabular}

To identify which geophysical parameters are useful to the prediction of CERES TOA SW anisotropy over each surface type and in each scattering direction, we applied two variable selection methods that efficiently explore variable combinations:

1) Random forest (RF) regression: Based on repeatedly drawn random subsets of geophysical parameters, this methods generates multiple decision trees (i.e., an RF) to predict TOA SW anisotropy. A subsequent permutation test measures each parameter's importance to the RF and allows for a threshold-based inclusion into or exclusion from an optimal subset.

2) Multiple linear regression (MLR): Geophysical parameters serve as regressors to predict TOA SW anisotropy. To avoid testing all possible combinations of parameter subsets, we use the genetic algorithm (GA). Starting out from several random subsets, the GA produces an optimal final subset.

Both methods are described in more detail in section $2 \mathrm{~d}$. The two optimal subsets, which both methods produce using single-surface footprints over each surface type and in each scattering direction, are compared and summarized in Table 3.

Parameters relating to a scene's brightness (normalized radiance) as well as general illumination and viewing geometry $\left(\theta_{S}, \varphi\right.$, and $\left.\theta_{V}\right)$ were consistently selected with the exception of fresh snow cases. As for general bidirectional reflectance distribution effects, the hotspot parameter was consistently selected over land surfaces free of fresh snow, while sun-glint reflectance was consistently selected only over open water surfaces as well as sea ice. Selection through the GA tended to include both hotspot parameter and sun-glint reflectance over almost all surface types and scattering directions, while selection through RF usually included either hotspot parameter or sun-glint reflectance.

Climatological AOD-potentially explaining atmospheric scattering through aerosol-appears consistently relevant over most surface types with the exception of fresh snow, sea ice, and nadir-directed scattering over forest, savannah, grassland, and open water. Gaseous absorption through atmospheric water vapor or ozone was sparsely selected by both methods with no relevance over fresh snow surfaces. For permanent snow and sea ice cases, on the other hand, both methods reported importance for at least two out of three scattering directions.

MCD43GB-based land surface anisotropy—which captures local and climatological bidirectional reflection effects perceived from a satellite perspective and is available in VIS and NIR as well as under white-sky and black-sky 
conditions - was frequently selected by both methods. For fresh snow cases we found less agreement or even mutual exclusion (at forward scattering). Parameters from ERA20C - which characterize the land surface state beyond climatological surface anisotropy-were collectively excluded for many surface types. For at least two out of three scattering directions, we found consistent relevance for high-vegetation LAI over forest, surface roughness and low-vegetation LAI over savannah, and 10-m wind over permanent snow cases.

We believe that potential changes in land surface appearance mostly followed a regular annual cycle and were therefore generally covered by above MCD43GF-based land surface anisotropy. Reasons for the apparent importance of ERA-20C parameters could be rooted in nonclimatological surface conditions: for example, irregular vegetation on/offsets over forest and savannah regions as well as wind-driven formation of unusual "sastrugi" over permanent snow (e.g., Corbett and Su 2015). Alternatively, LAI (of low or high vegetation) was potentially selected due to its correspondence to NDVI, which served to stratify into separate CERES ADMs (see section 2a).

Apart from fresh snow cases, we found a general agreement of both methods on inclusion or exclusion of parameters. We interpret that a layer of fresh snow alienates the surface anisotropy sufficiently to render MCD43GF-based surface anisotropy useless. Interestingly, the GA selected high-vegetation LAI as important. We suspect that snow-covered forests might appear different enough in anisotropy compared to plain snow fields, leading to the selection of this parameter.

To further understand parameter selection, we plotted coefficients of determination, $R^{2}$ [as defined in Wilks (2005)], in Table 4. Coefficients are determined either by multiple linear regression using the optimal set from GA selection (top row) or linear regression using individual parameters with TOA anisotropy (rows below). The $R^{2}$ of optimal models reach highest values $(\geq 0.85)$ above permanent snow, desert, and open water surfaces, as well as for backward- and nadir-scattering directions over vegetated land surfaces (forest, savannah, grassland, and shrubland). Corresponding forward-scattering directions showed lower $R^{2}$ (by up to 0.4 compared against backward and nadir). We suspect that shadows cast by vegetation (mostly perceived in the forward-scattering direction) facilitate most complex anisotropy variations. We found low $R^{2}$ above fresh snow surfaces. Looking at the predictive power of individual variables (second row and below in Table 4) across surface types we noticed that most variables in the GA optimal set (highlighted in bold) have higher $R^{2}$ than unselected variables. Exceptions with a high $R^{2}$ and yet no selection for the optimal set (such as $R_{w}^{\mathrm{NIR}}$ in the nadir-observed desert cases with an $R^{2}$ of 0.64 ) could imply that an alternative variable (or a combinations of other variables) explain the anisotropy variability better and thus make this variable obsolete. Exceptions which are selected for the optimal set despite a low $R^{2}$ (such as the LAI of high vegetation in fresh snow surfaces with $R^{2} \leq 0.001$ ) could occur if the variable is part of a superior combination of variables or when rare circumstances (such as vegetation emerging from a snow cover) require this variable to indicate anisotropy changes. Some parameters explain anisotropy variations exceptionally well. The hotspot effect aids backward- and nadir-scattering directions over land surface types (usually $R^{2}>0.6$ ). In some cases it outperforms BOA anisotropies derived from the RossThick-LiSparse model. The sun-glint reflectance predicts well for nadir- and forward-scattering directions above open water $\left(R^{2}\right.$ of 0.85 and 0.70 , respectively), and appears more suitable than $10-\mathrm{m}$ wind speed (0.001 and 0.026 correspondingly). Possible reasons for the methods selecting variables differently are discussed in section 4.

After identification of optimal parameter subsets through two different selection methods, we can use these subsets as input to train radiance-to-flux converting ANNs (section 2e). The use of different input sets potentially affects the ability to generalize conversion, that is, to predict TOA SW fluxes of unseen test data (see section 2c). Shown by surface type and scattering direction, Table 5 compares test errors of different input sets: optimal subsets from both selection methods (labeled by their method) and two extreme subsets-all available parameters (listed as "all parameters," only excluding parameters marked as " $/$ " in Table 3) and viewingillumination geometry parameters only ("minimum,", including normalized radiance, $\theta_{S}, \varphi$, and $\left.\theta_{V}\right)$. Compared to CERES flux estimates, we generally found poorest performance for the minimum input set [set (iv)], on average $0.52-1.08 \mathrm{~W} \mathrm{~m}^{-2}$ worse than the best performer. Using the complete parameter set [set (iii)] slightly surpassed optimal parameter sets [sets (i) and (ii)] for particular surfaces like sea ice cases (by up to $0.47 \mathrm{~W} \mathrm{~m}^{-2}$ ) and forward- and nadir-scattered light over savannah cases (by up to $1.39 \mathrm{~W} \mathrm{~m}^{-2}$ ). However, we found optimal sets to exceed the performance of the complete parameter set in other cases even more distinctly (e.g., nadir scattering over permanent snow and desert by a difference of 0.8 and $1.97 \mathrm{~W} \mathrm{~m}^{-2}$, respectively).

Generally large flux errors over fresh snow surfaces (up to $9.98 \mathrm{~W} \mathrm{~m}^{-2}$ ) were due to large absolute values (a product of snow's high albedo in lower latitudes with correspondingly low $\theta_{S}$ ) and the apparent lack of suitable 


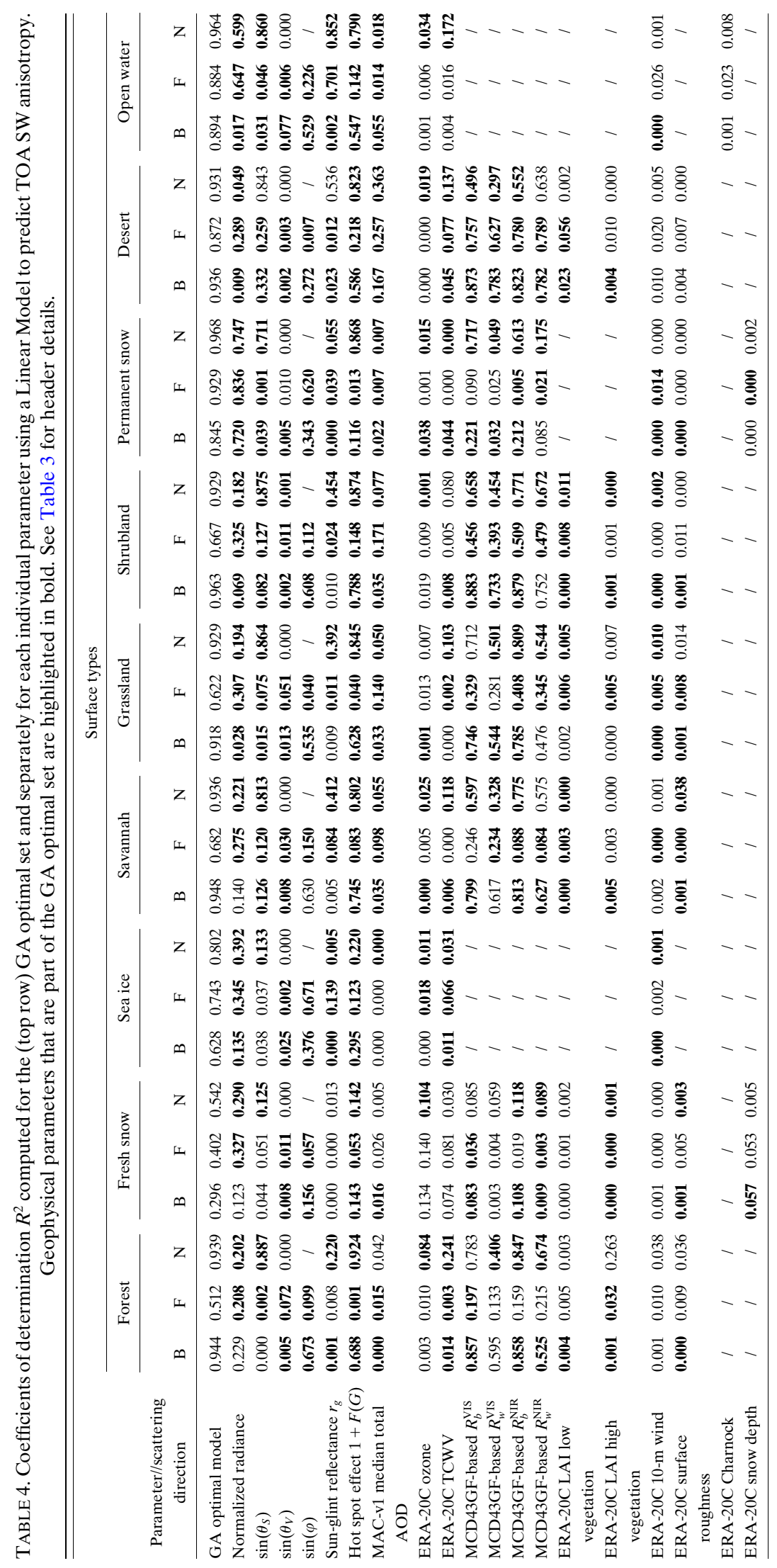




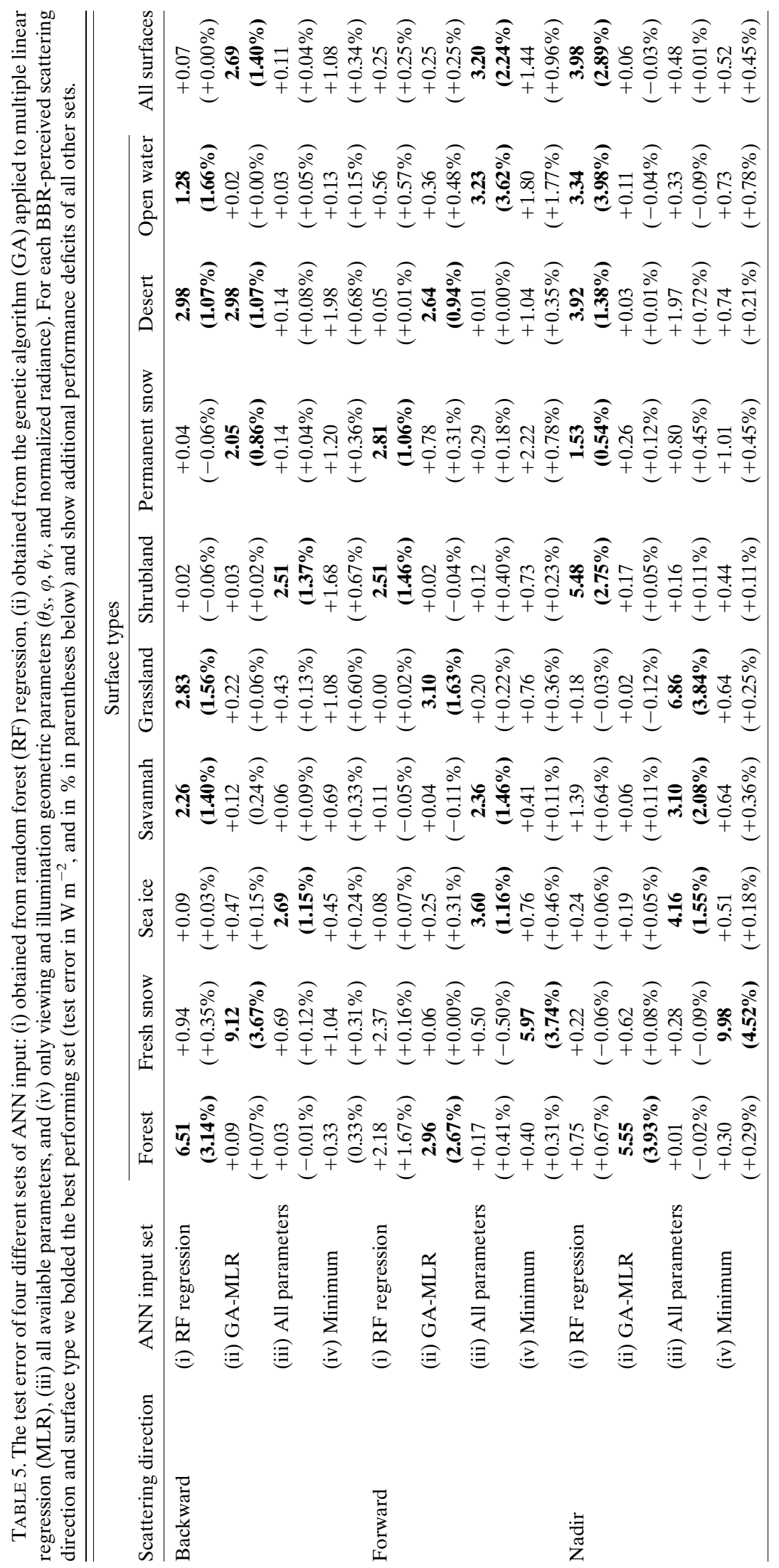




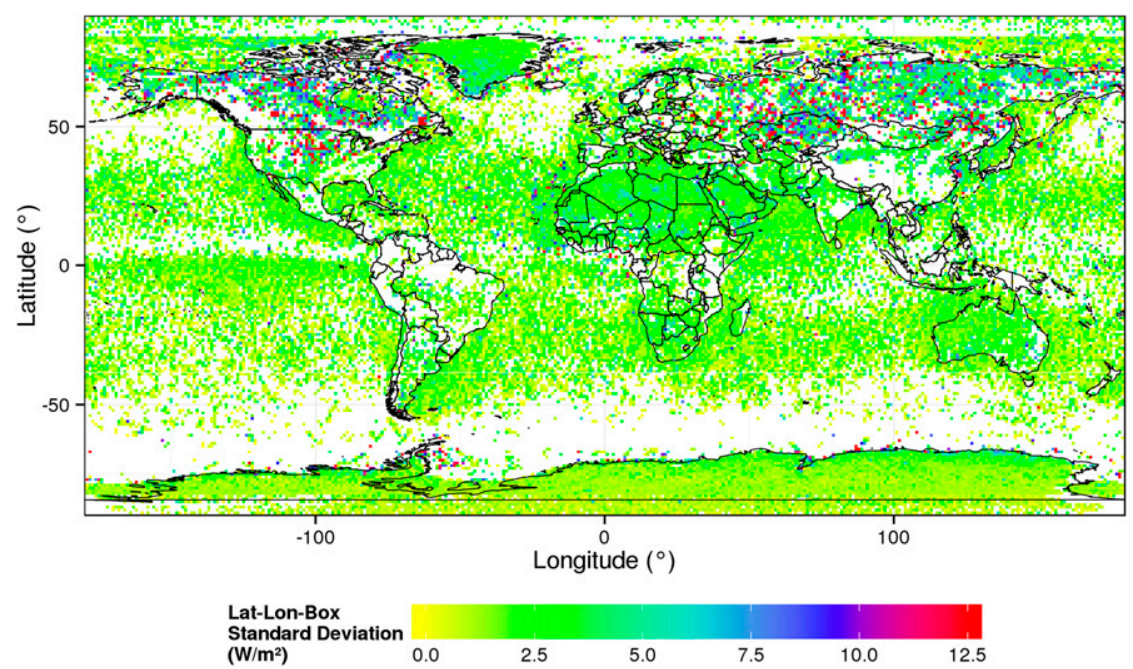

FIG. 2. Applied to the test dataset (introduced in section 2c), we present error standard deviations of ANN-predicted [using set (i), as listed in Table 5] clear-sky TOA SW Fluxes of single-surface CERES footprints within $1^{\circ} \times 1^{\circ}$ latitude-longitude boxes. Individual footprints are either at nadir or $55^{\circ} \theta_{V}$, aiming for a BBR-like viewing geometry.

candidates relating to TOA anisotropy (cf. Table 3). In effect, the minimum input [set (iv)] performed best in two out of three scattering directions.

For all other surface types, the large performance gap between minimum and all other parameter subsets revealed the added value of collocated auxiliary parameters. The overall small performance difference between optimal subsets and the full set of parameters is discussed in section 4. Averaged over all surfaces, we observed little performance difference between optimal sets (on average $0.00-0.07 \mathrm{~W} \mathrm{~m}^{-2}$ ). For further error analysis, we focused on ANN results that used set (i) as input.

Figure 2 shows a global map of deviations in ANN predictions from original CERES flux estimates. Regions in northern latitudes exceeding the mission requirement of $10 \mathrm{~W} \mathrm{~m}^{-2}$ could largely be attributed to fresh snow cases, while larger uncertainties near Antarctica were caused by sea ice. Empty areas (e.g., the Southern Ocean) were either persistently cloudy or footprints composed of multiple surfaces (see section 3c).

Figure 3 presents which angular combinations $\left(\theta_{S}\right.$ and $\varphi)$ caused ANN predictions to deviate from CERES estimates. We found that uncertainties increased near the boundaries of sampled angles (e.g., shrubland), at lower $\theta_{S}$ (e.g., fresh snow), and around pronounced anisotropy (such as the sun-glint region over open water). The angular space that will be observed by EarthCARE's BBR instrument (shown in gray shade; ESA 2015, communication) was only partly covered by CERES samples, the result of a difference in equator crossing time between EarthCARE (1400 LT) and Aqua (1330 LT) or Terra (1030 LT). To test how well ANNs predicted anisotropies over unseen angular combinations, we manually excluded two regions $\left(15^{\circ} \leq \varphi \leq 25^{\circ}\right.$ and $155^{\circ} \leq \varphi \leq 165^{\circ}$ at $\left.0^{\circ} \leq \theta_{S} \leq 80^{\circ}\right)$. Retrained ANNs using remaining samples were applied over excluded regions. Compared against a training using complete data, the increase in test error was well below $1.5 \mathrm{~W} \mathrm{~m}^{-2}$ over most surface types. Only open water (an average increase in excluded regions of $3.6 \mathrm{~W} \mathrm{~m}^{-2}$ or $4.48 \%)$, grasslands $\left(2.7 \mathrm{~W} \mathrm{~m}^{-2}\right.$ or $\left.1.56 \%\right)$, sea ice $\left(2.5 \mathrm{~W} \mathrm{~m}^{-2}\right.$ or $1.01 \%)$, fresh snow $\left(2.1 \mathrm{~W} \mathrm{~m}^{-2}\right.$ or $\left.0.92 \%\right)$, and permanent snow $\left(1.9 \mathrm{~W} \mathrm{~m}^{-2}\right.$ or $\left.0.71 \%\right)$ cases demonstrated a larger increase in excluded angular portions (shown in Fig. 4). We attribute the large increase over open water to the partial exclusion of dominant sun-glint portions. Interestingly, the performance of some (nonexcluded) angular combinations (like open water $50^{\circ} \leq \varphi \leq 70^{\circ}$ and fresh snow $70^{\circ} \leq \varphi \leq 120^{\circ}$ ) profited from ANN training using a reduced training set. This suggests that further subdivision in angular space (beyond forward and backward scattering) allows for better performance.

\section{c. Modifications for EarthCARE}

To apply above ANN-based anisotropy prediction operationally, dynamic surface types (fresh snow and sea ice) need to be consistently assigned to CERES surface classes. Instead of relying on CERES-reported surface fractions (as done in sections $3 a$ and $3 b$ for single-surface footprints), we delineated sea ice from ice-free ocean using ERA-20C sea ice fraction (greater than zero). The best candidate to separate snow-free from fresh snow or permanent snow cases was ERA-20C snow depth (greater than zero or greater than $4.9 \mathrm{~m}$, respectively). Naturally, snow depth fails to report the 


\section{Performance of ANN-Predicted Clear-Sky TOA SW Fluxes on Test Data}

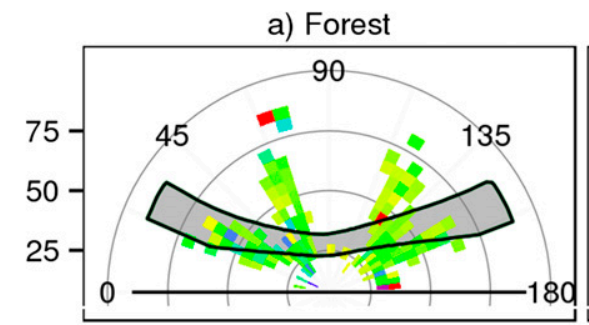

b) Fresh Snow

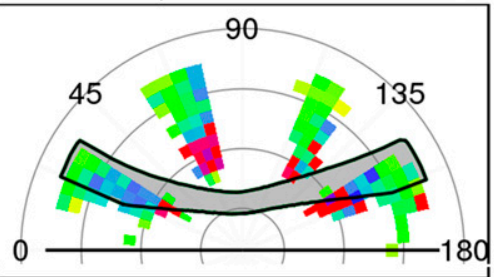

e) Grass

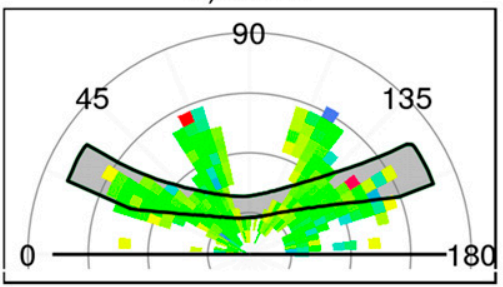

h) Desert

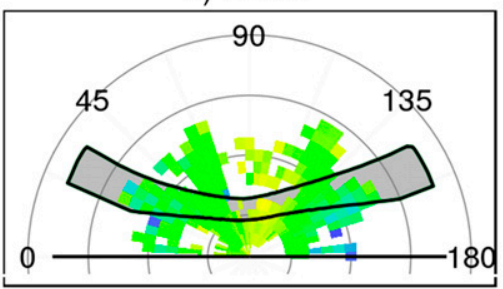

c) Sea Ice

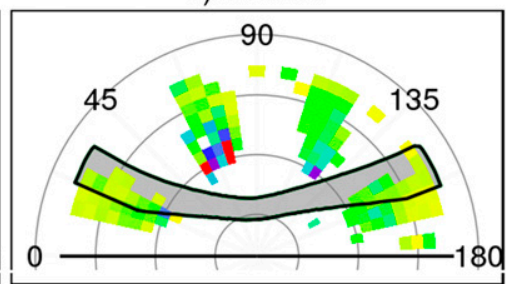

f) Shrubland

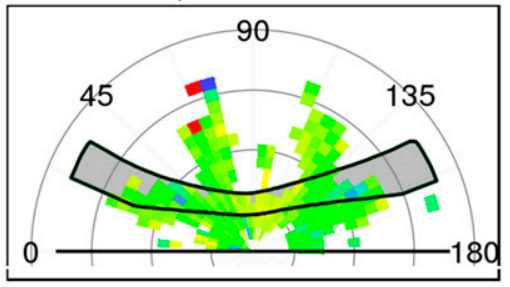

i) Open Water

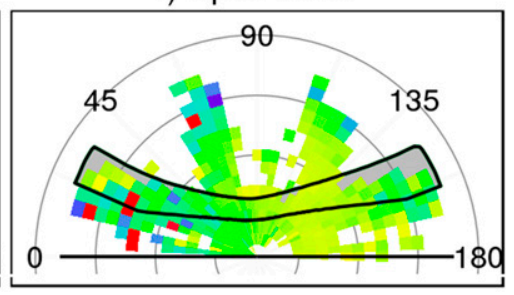

Relative Azimuth Angle $\left({ }^{\circ}\right)$

\section{Angular Bin Standard Deviation $\left(\mathrm{W} / \mathrm{m}^{2}\right)$}

0.0
2.5

5.0
7.5

10.0

FIG. 3. Standard deviations of ANN-predicted [using set (i), as shown in Table 5] clear-sky TOA SW Fluxes of single-surface CERES footprints of the test dataset from original CERES SSF 4 estimates within $5^{\circ} \times 5^{\circ}$ angular boxes of $\theta_{S}$ and $\varphi$. Individual footprints are at $55^{\circ}$ $\theta_{V}$, aiming for a BBR-like off-nadir-viewing geometry. The gray-shaded area highlight expected angular combination seen by EarthCARE's BBR when sampling globally.

area covered by snow and only indicates snow contamination in a footprint. Compared to CERES classes, we identified the partial presence of sea ice, fresh snow, and permanent snow with hit rates (and false alarm rates) of 97.1\% (8.5\%), 92.9\% (42.1\%), and 99.6\% (0.5\%), respectively. We suspect that large false alarm rates over fresh snow resulted from ERA-20C's coarse resolution (approximately 125-km grid spacing). During the EarthCARE mission ECMWF forecasts (so-called X-MET, using IFS cy41r2) will provide information instead of ERA-20C. Reported fields will have a finer spatial resolution (approximately 8-km grid spacing) and potentially produce higher hit (and lower false alarm) rates.

To further assure operational fitness, we verified that anisotropy predictions over mixed surfaces (roughly a third of all obtained CERES footprints) satisfied mission requirements. To predict a TOA $\mathrm{SW}$ anisotropy $\mathbb{R}_{\text {mix }}$ over a footprint containing several surface types, we used the approach by Bertrand et al. (2005), shown in Eq. (16). Here, $\mathbb{R}_{1}$ and $\mathbb{R}_{2}$ denote predicted anisotropies over each surface type, and $f_{1}$ and $f_{2}$ respective surface fraction:

$$
\mathbb{R}_{\text {mix }}=\frac{f_{1} \mathbb{R}_{1} \bar{a}_{1}+f_{2} \mathbb{R}_{2} \bar{a}_{2}}{f_{1} \bar{a}_{1}+f_{2} \bar{a}_{2}} .
$$

Required surface type albedos, $\bar{a}_{1}$ and $\bar{a}_{2}$, were approximated from single-surface footprints of similar latitude (categorized into tropical zone: $23.5^{\circ} \mathrm{S}-23.5^{\circ} \mathrm{N}$; midlatitudes: $23.6^{\circ}-66.5^{\circ} \mathrm{N} / \mathrm{S}$; and polar regions $66.6^{\circ}-$ $90^{\circ} \mathrm{N} / \mathrm{S}$ ) and solar zenith angle (by increments of $5^{\circ}$ from $10^{\circ}$ to $85^{\circ} \theta_{S}$ ). We found that the above methodology worked reliably for snow- and ice-free mixtures: flux uncertainties were of similar magnitude (not shown) as single-surface footprints (Table 5). Only two 


\section{Performance Change after ANN-Training using Incomplete Data}

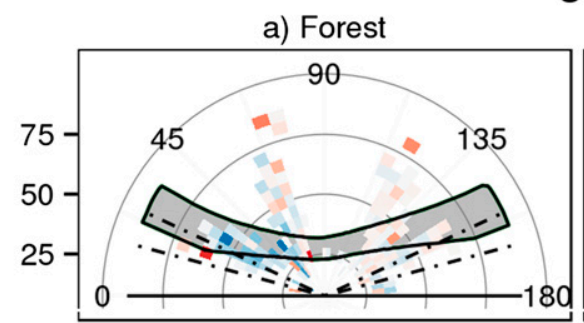

b) Fresh Snow

c) Sea Ice
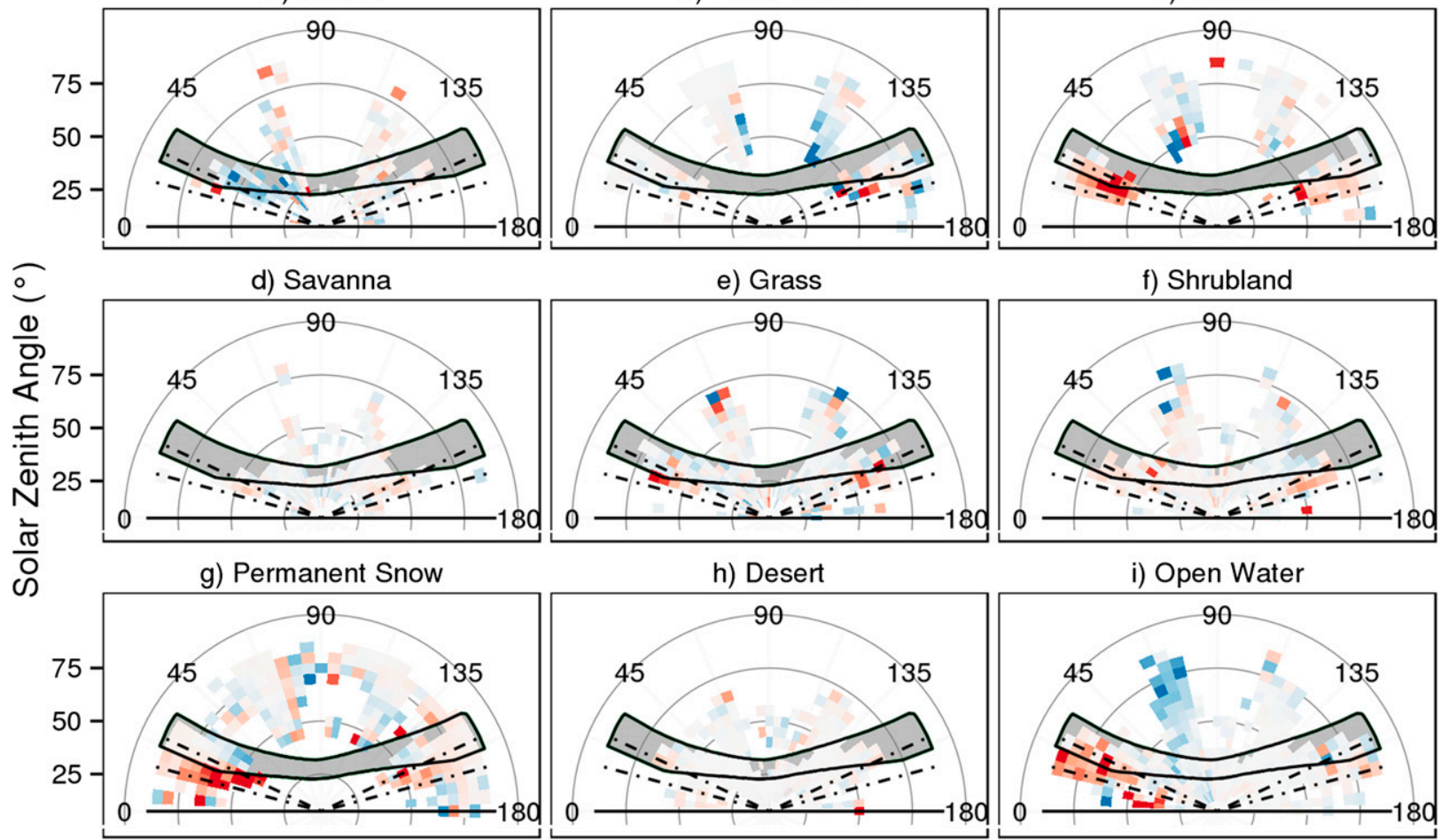

Relative Azimuth Angle $\left({ }^{\circ}\right)$
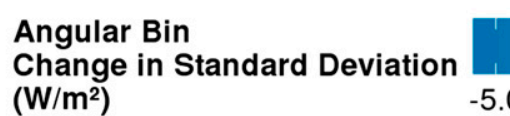

$-5.0$

$-2.5$

0.0

2.5

5.0

FIG. 4. Repeated ANN training using an incomplete training dataset (excluding angular portions of $\varphi \in 15^{\circ}-25^{\circ}, \varphi \in 155^{\circ}-165^{\circ}$, and $\theta_{S} \in 0^{\circ}-80^{\circ}$, highlighted by dashed lines), we present changes in test error standard deviations of ANN-predicted clear-sky TOA SW fluxes compared against an ANN training using all available data (shown in Fig. 3).

combinations caused flux uncertainties larger than $10 \mathrm{~W} \mathrm{~m}^{-2}$ : grassland mixed with desert at nadir view with $17.84 \mathrm{~W} \mathrm{~m}^{-2}$ (2708 footprints or $0.07 \%$ of all nadir cases) and shrubland mixed with open water at nadir view with $26.04 \mathrm{~W} \mathrm{~m}^{-2}$ (14823 footprints or $0.37 \%$ ). Assuming we had accurate information on snow fraction, mixtures including snow or ice produced large uncertainties across observed surface pairings (fresh snow: 16.1-194.1 $\mathrm{W} \mathrm{m}^{-2}$; permanent snow: $15.6-150.2 \mathrm{~W} \mathrm{~m}^{-2}$; and sea ice: $15.2-59.1 \mathrm{~W} \mathrm{~m}^{-2}$ ), particularly for combinations including stark albedo contrasts, like with open water. ERA-20C sea ice fraction had limited correspondence with CERES-reported fractions (a Pearson correlation coefficient of 0.78 ) and reported usually higher values than CERES (biased by $8.2 \%$ ). We suspect that the poor agreement was again due to ERA-20C's coarse resolution and would have resulted in even larger flux prediction errors.
Due to our inability to determine surface fractions of fresh or permanent snow and the large uncertainty above mixtures with snow or ice, we treated footprints with any snow or ice contamination as single-surface footprints of respective snow or ice type. We extended training as well as the test set, and repeated ANN training. For fresh snow and sea ice which contained a large fraction of mixed footprints $(17.5 \%$ and $74.2 \%$ of snow and ice footprints were mixed, respectively) we discovered that a simpler input set [set (iv)] resulted in lower flux uncertainties compared to set (i). Prediction over permanent snow (only $1.3 \%$ of permanent snow footprints were mixed) remained optimal using set (i). Application of retrained ANNs on extended test data resulted in higher flux uncertainties (Table 6) than a pure surface training and testing (cf. Table 5), clearly the result of introduced heterogeneity of mixed-surface footprints into ANN training and testing. 
TABLE 6. Test errors (in $\mathrm{W} \mathrm{m}^{-2}$ ) after inclusion of mixed footprints containing fresh snow, sea ice, or permanent snow into training and test sets. Sets (i) and (iv) correspond to input sets shown in Table 5. Expected performances for all other surface types are listed in Table 5 [set (i)].

\begin{tabular}{llll}
\hline \hline & \multicolumn{3}{c}{ Surface types } \\
\cline { 2 - 4 } & Fresh snow & Sea ice & Permanent snow \\
\hline ANN input set & Set (iv) & Set (iv) & Set (i) \\
Fraction of mixed & $17.5 \%$ & $74.2 \%$ & $1.3 \%$ \\
$\quad$ footprints & & & \\
Backward & 11.64 & 5.41 & 2.75 \\
& $(5.91 \%)$ & $(2.51 \%)$ & $(1.06 \%)$ \\
Forward & 8.27 & 9.63 & 4.04 \\
& $(6.33 \%)$ & $(7.01 \%)$ & $(1.55 \%)$ \\
Nadir & 14.62 & 8.06 & 2.64 \\
& $(8.83 \%)$ & $(4.88 \%)$ & $(1.14 \%)$ \\
\hline
\end{tabular}

\section{Discussion}

The SW radiance-to-flux conversion over clear-sky scenes is challenging due the spatial heterogeneity and temporal variation of factors driving bidirectional reflectance distribution at TOA. The CERES solution for land surfaces involved separate ADMs per regional and temporal bin, and further separation through levels of surface-specific parameters (such as NDVI, surface elevation variability, or fractional coverage through snow or ice). We have successfully shown that the use of geophysical parameters-serving to substitute CERES-utilized ones during the EarthCARE mission or expanding the list by using so-far unaccounted sources-could explain variations across bins, and thus help to dissolve binning. We presented the identification of optimal subsets through the use of two variable selection methods (i.e., random forest regression followed by a permutation test and multiple linear regression in combination with the genetic algorithm), applied per surface type and scattering direction as perceived by EarthCARE's BBR. We then analyzed the use of optimal parameter subsets as input to radianceto-flux converting ANNs. To ensure mission-worthy flux estimates we presented modifications concerning footprints partly covered in snow or ice.

Even though we applied two very different variable selection methods, we mostly found agreement on in- or exclusion of parameters. Both the selection of the hotspot parameter over land surfaces free of fresh snow and the selection of sun-glint reflectance over open water confirm our understanding of general bidirectional reflectance effects established at Earth's surface. The additional and consistent selection of MCD43GF-based surface anisotropy confirms that local climatological surface conditions further impact such effects over all land types free of fresh snow. Apart from fresh snow scenes, AOD — even though extracted from a climatology-was frequently selected, we presume due to reoccurring spatial patterns like dust storms or biomass-burning events which impact TOA SW anisotropy through scattering or absorption. Atmospheric gases (here total column water vapor and ozone) from ERA-20C, which could likewise impact TOA SW anisotropy through absorption, were only occasionally chosen. We found their selection foremost over sea ice and permanent snow surfaces, presumably because only these regions have a large enough variability in respective gas concentration. The identification of ERA-20C LAI of low and high vegetation over savannah and forest surfaces, respectively, could further support the importance of land surface anisotropy. On the other hand, their selection could simply be a response to CERES-based stratification by NDVI. Further parameters that directly reflect the CERES methodology and which were identified by applied variable selection methods are AOD and sun-glint reflectance (using 10-m wind speed) over open water.

As with any alternative statistical tool, employed methods for variable selection could potentially choose meaningless variables. Noisy data, unrepresentative validation sets (used to select one optimal set over the other), or a method's tendency to include more or fewer variables are all factors that make some variable selections controversial. We suspect that genetic algorithms had a tendency to include more parameters: the method persistently identified an importance of sun-glint reflectance over land and the hotspot effect over water, both of which we find questionable. Further reasons for inconsistent parameter selection could stem from fundamental differences with respect to regression (linear vs nonlinear), selection strategy (performance based vs threshold based), and the vast use of random numbers (to produce parameter subsets vs to construct decision trees) when comparing multiple linear regression in combination with genetic algorithms and random forest with subsequent permutation testing. ANN training, while unable to eliminate useless input parameters altogether, can reduce their weight to near zero. We believe that such reduction of neural weight caused ANNs using all parameters versus optimal parameter subsets to perform similarly on unseen test data. In turn, identifying optimal parameters had only limited impact on ANN performance and mainly helped to understand TOA anisotropy driving factors as captured by CERES ADMs.

The performance of ANNs indicates that we could successfully reproduce CERES models. ANN's overall relative errors-though covering only a portion of the viewing zenith angular space-were near the CERESdefined goal of $2.3 \%$ over ocean, $1.6 \%$ over land, and $2.0 \%$ over snow and ice (Su et al. 2015b).

TOA SW anisotropy over fresh snow remained largely unexplained. Future efforts should continue to explore sources characterizing anisotropy fluctuations 
reaching TOA. Radiative transfer simulations should also clarify the role of different aerosol types in altering TOA SW anisotropy aloft different types of surfaces.

Acknowledgments. We thank Nicolas Clerbaux, Howard W. Barker, Tobias Wehr, and all other members of the CLARA team for helpful discussion, Maximilian Voigt, Nicole Docter, and Rene Preusker for technical support, and to the Atmospheric Sciences Data Center at the National Aeronautics and Space Administration Langley Research Center for providing the Clouds and the Earth's Radiant Energy System Single Scanning Footprint TOA/Surface Fluxes and Clouds data product. Furthermore, we thank anonymous reviewers for their constructive comments. This work was possible through funding within the ESA Contract 4000112019/14/NL/CT.

\section{REFERENCES}

Barker, H. W., M. P. Jerg, T. Wehr, S. Kato, D. P. Donovan, and R. J. Hogan, 2011: A 3D cloud-construction algorithm for the EarthCARE satellite mission. Quart. J. Roy. Meteor. Soc., 137, 1042-1058, https://doi.org/10.1002/qj.824.

Bertrand, C., N. Clerbaux, A. Ipe, S. Dewitte, and L. Gonzalez, 2005: Angular distribution models, anisotropic correction factors, and mixed clear-scene types: A sensitivity study. IEEE Trans. Geosci. Remote Sens., 43, 92-102, https://doi.org/ 10.1109/TGRS.2004.838361.

Breiman, L., 2001: Random forests. Mach. Learn., 45, 5-32, https://doi.org/10.1023/A:1010933404324.

Caldwell, M. E., and Coauthors, 2017: The EarthCARE mission BBR instrument: Ground testing of radiometric performance. Proc. SPIE, 10402, 1040204, https://doi.org/10.1117/12.2273148.

Capderou, M., and M. Viollier, 2006: True along-track scan to improve radiation budget estimations. J. Atmos. Oceanic Technol., 23, 1093-1103, https://doi.org/10.1175/JTECH1907.1.

Charnock, H., 1955: Wind stress on a water surface. Quart. J. Roy. Meteor. Soc., 81, 639-640, https://doi.org/10.1002/qj.49708135027.

Corbett, J., and W. Su, 2015: Accounting for the effects of sastrugi in the CERES clear-sky Antarctic shortwave angular distribution models. Atmos. Meas. Tech., 8, 3163-3175, https://doi.org/10.5194/amt-8-3163-2015.

Cox, C., and W. Munk, 1954: Measurement of the roughness of the sea surface from photographs of the sun's glitter. J. Opt. Soc. Amer., 44, 838-850, https://doi.org/10.1364/JOSA.44.000838.

Dewitte, S., L. Gonzalez, N. Clerbaux, A. Ipe, C. Bertrand, and B. D. Paepe, 2008: The Geostationary Earth Radiation Budget edition 1 data processing algorithms. Adv. Space Res., 41, 1906-1913, https://doi.org/10.1016/j.asr.2007.07.042.

Domenech, C., and T. Wehr, 2011: Use of artificial neural networks to retrieve TOA SW radiative fluxes for the EarthCARE mission. IEEE Trans. Geosci. Remote Sens., 49, 1839-1849, https://doi.org/10.1109/TGRS.2010.2102768.

Illingworth, A. J., and Coauthors, 2015: The EarthCARE satellite: The next step forward in global measurements of clouds, aerosols, precipitation and radiation. Bull. Amer. Meteor. Soc., 96, 1311-1332, https://doi.org/10.1175/BAMS-D-12-00227.1.

Kinne, S., and Coauthors, 2013: MAC-v1: A new global aerosol climatology for climate studies. J. Adv. Model. Earth Syst., 5, 704-740, https://doi.org/10.1002/jame.20035.
Loukachine, K., and N. G. Loeb, 2004: Top-of-atmosphere flux retrievals from CERES using artificial neural networks. Remote Sens. Environ., 93, 381-390, https://doi.org/10.1016/j.rse.2004.08.005.

Lucht, W., C. B. Schaaf, and A. H. Strahler, 2000: An algorithm for the retrieval of albedo from space using semiempirical BRDF models. IEEE Trans. Geosci. Remote Sens., 38, 977-998, https://doi.org/10.1109/36.841980.

Mehmood, T., K. H. Liland, L. Snipen, and S. Sæbø, 2012: A review of variable selection methods in partial least squares regression. Chemom. Intell. Lab. Syst., 118, 62-69, https://doi. org/10.1016/j.chemolab.2012.07.010.

Moody, E. G., M. D. King, C. B. Schaaf, and S. Platnick, 2008: MODIS-derived spatially complete surface albedo products: Spatial and temporal pixel distribution and zonal averages. J. Appl. Meteor. Climatol., 47, 2879-2894, https://doi.org/ 10.1175/2008JAMC1795.1.

Poli, P., and Coauthors, 2016: ERA-20C: An atmospheric reanalysis of the twentieth century. J. Climate, 29, 4083-4097, https://doi.org/10.1175/JCLI-D-15-0556.1.

Rahman, H., B. Pinty, and M. M. Verstraete, 1993: Coupled surface-atmosphere reflectance (CSAR) model: 2. Semiempirical surface model usable with NOAA Advanced Very High Resolution Radiometer data. J. Geophys. Res., 98, 20 791-20 801, https://doi.org/10.1029/93JD02072.

Ross, J., 1981: The Radiation Regime and Architecture of Plant Stands. 1st ed. Dr. W. Junk Publishers, 391 pp.

Scrucca, L., 2013: GA: A package for genetic algorithms in R. J. Stat. Software, 53, 4, https://doi.org/10.18637/jss.v053.i04.

Strobl, C., and A. Zeileis, 2009: Party on!-A new, conditional variable importance measure for random forests available in party. Tech. Rep. 50, University of Munich Department of Statistics, 5 pp.

Su, W., J. Corbett, Z. Eitzen, and L. Liang, 2015a: Next-generation angular distribution models for top-of-atmosphere radiative flux calculation from CERES instruments: Methodology. Atmos. Meas. Tech., 8, 611-632, https://doi.org/10.5194/amt-8-611-2015.

,,,--- and $-2015 \mathrm{~b}$ : Next-generation angular distribution models for top-of-atmosphere radiative flux calculation from CERES instruments: Validation. Atmos. Meas. Tech., 8, 3297-3313, https://doi.org/10.5194/amt-8-3297-2015.

Theodoridis, S., and K. Koutroumbas, 2009: Pattern Recognition. 4th ed. Academic Press, 984 pp.

Townshend, J. R. G., Ed., 1992: Improved global data for land applications. International Geosphere-Biosphere Programme Tech. Rep. 20, 45 pp.

Viollier, M., C. Standfuss, O. Chomette, and A. Quesney, 2009: Top-of-atmosphere radiance-to-flux conversion in the SW domain for the ScaRaB-3 instrument on Megha-Tropiques. J. Atmos. Oceanic Technol., 26, 2161-2171, https://doi.org/ 10.1175/2009JTECHA1264.1.

Wald, L., and J. M. Monget, 1983: Sea surface winds from sun glitter observations. J. Geophys. Res., 88, 2547-2555, https://doi.org/10.1029/JC088iC04p02547.

Wallace, K., N. Wright, D. Spilling, K. Ward, and M. Caldwell, 2009: The broadband radiometer on the EarthCARE spacecraft. Proc. SPIE, 7453, 74530H, https://doi.org/10.1117/12.825837.

Wielicki, B. A., B. R. Barkstrom, E. F. Harrison, R. B. Lee III, G. L. Smith, and J. E. Cooper, 1996: Clouds and the Earth's Radiant Energy System (CERES): An Earth Observing System experiment. Bull. Amer. Meteor. Soc., 77, 853-868, https:// doi.org/10.1175/1520-0477(1996)077<0853:CATERE >2.0.CO;2.

Wilks, D. S., 2005: Statistical Methods in the Atmospheric Sciences. 2nd ed. Elsevier, 648 pp. 